\title{
The Association between Phase Angle of Bioelectrical Impedance Analysis and Survival Time in Advanced Cancer Patients: Preliminary Study

\section{So Yeon Lee, Yong Joo Lee ${ }^{1}$, Jung-Hwa Yang, Chul-Min Kim, Whan-Seok Choi*}

Departments of Family Medicine and ${ }^{1}$ Palliative Medicine, Seoul St. Mary's Hospital, The Catholic University of Korea College of Medicine, Seoul, Korea

Background: A frequent manifestation of advanced cancer patients is malnutrition, which is correlated with poor prognosis and high mortality. Bioelectrical impedance analysis (BIA) is an easy-to-use and non-invasive technique to evaluate changes in body composition and nutritional status. We investigated BIA-derived phase angle as a prognostic indicator for survival in advanced cancer patients.

Methods: Twenty-eight patients treated at the hospice center of Seoul St. Mary's Hospital underwent BIA measurements from January, 2013 to May, 2013. We also evaluated palliative prognostic index (PPI) and palliative performance scale to compare with the prognostic value of phase angle. Cox's proportional hazard models were constructed to evaluate the prognostic effect of phase angle. The Kaplan Meier method was used to calculate survival.

Results: Using univariate Cox analysis, phase angle (hazard ratio [HR], 0.61/per degree increase; 95\% confidence interval [CI], 0.42 to $0.89 ; \mathrm{P}=0.010)$, PPI (HR, 1.21; 95\% CI, 1.00 to $1.47 ; \mathrm{P}=0.048$ ) were found to be significantly associated with survival. Adjusting age, PPI, body mass index, phase angle significantly showed association with survival in multivariate analysis (HR, 0.64/per degree increase; $95 \% \mathrm{CI}, 0.42$ to $0.95 ; \mathrm{P}=0.028$ ). Survival time of patients with phase angle $\geq 4.4^{\circ}$ was longer than patients with phase angle $<4.4^{\circ}(\log$ rank, 6.208; P-value $=0.013)$.

Conclusion: Our data suggest BIA-derived phase angle may serve as an independent prognostic indicator in advanced cancer patients.

Keywords: Electric Impedance; Neoplasms; Palliative Care; Prognosis

\section{INTRODUCTION}

Received: August 26, 2013, Accepted: June 16, 2014

${ }^{*}$ Corresponding Author: Whan-Seok Choi

Tel: +82-2-2258-6277, Fax: +82-2-2258-1903

E-mail: fmchs@catholic.ac.kr

Korean Journal of Family Medicine

Copyright (C) 2014 The Korean Academy of Family Medicine

(a) This is an open-access article distributed under the terms of the Creative Commons Attribution Non-Commercial License (http://creativecommons.org/licenses/by-nc/3.0) which permits unrestricted noncommercial use, distribution, and reproduction in any medium, provided the original work is properly cited.
Weight loss and malnutrition are common problem in cancer patients. It has been reported that $20 \%$ of cancer mortality is correlated with cancer anorexia-cachexia syndrome. ${ }^{1)}$ Poor prognosis is expected when patients have involuntary weight loss, anorexia, weakening muscle strength, and loss of muscle mass that are known as symptoms of cancer anorexia-cachexia syndrome. Malnutrition results in body fluid imbalance and cell membrane change. ${ }^{2)}$ Therefore, body composition analysis 
plays an important role in measuring nutrition status. ${ }^{3)}$ Classic objective nutritional status are measured by weight, muscle diameter, serum albumin, transferrin, and blood urea nitrogen (physical measurement or blood test). Recently invented bioelectrical impedance analysis (BIA) technique provides early nutritional status predictions by calculating cell membrane change and fluid imbalance. BIA performs noninvasive and fast analysis of body composition, body fluid balance, and cellular health status. BIA measure resistance and reactance via implanted sensors. Resistance is related to total body tissue fluid while reactance is associated with conserving cell membrane. ${ }^{4)}$ Phase angle is an item of BIA test which calculates cellular health status and nutritional status by resistance of body fluid and reactance of cellular membrane. While high phase angle score represents a good cellular membrane function, low phase angle is closely related to apoptosis of cell and decrease of cellular matrix compound. ${ }^{5)}$ The spectrum of BIA research is quite broad. BIA is also applied to prognosis study by nutritional status analysis. ${ }^{6}$

In previous studies using BIA, Gupta et al. ${ }^{7-10)}$ demonstrated prognostic value of phase angle in pancreatic cancer, lung cancer, breast cancer, and colorectal cancer patients. Santarpia et al. ${ }^{11)}$ showed association between Karnofsky index and phase angle in advanced cancer patients. Paiva et al. ${ }^{12)}$ also reported phase angle as an independent prognostic factor in a prospective observational study. Norman et al. ${ }^{13)}$ showed that phase angle lower than $50 \%$ in the standard reference was associated with decrease of physical function, nutritional status, and increase of mortality. However, the relationship between phase angle and survival in terminal cancer patients has never been studied in Korea. Therefore, the objective of this study was to evaluate the correlation between phase angle and survival in terminal cancer using phase angle of BIA test and the effect on inpatients of hospice and palliative unit.

\section{METHODS}

\section{Study Population}

We enrolled 28 hospice inpatients of the Seoul St. Mary' s Hospital, the Catholic University of Korea from January 1, 2013 to May 31, 2013. The inclusion criteria of the study were as follows: 1) inpatients over 18 years old who agreed to participate in the study; 2) inpatients who were diagnosed with terminal cancer. The exclusion criteria were as follows: 1) patients who were impossible to communicate with this study (ritual deterioration, severe cognitive impairment); 2) patients who have lymphedema of upper or lower extremity; 3 ) patients who have history of edema (heart failure, liver failure, inferior vena cava syndrome, superior vena cava syndrome); 4) patients using diuretic on medical record; 5) patients with ascites, pleural effusions, and pericardial effusion.

\section{Study Methods}

This study used a prospective observational study method analyzing the associations between phase angle and the survival time in terminal cancer patients by using BIA.

\section{1) Demographic and clinical information}

Demographic and clinical data of age, gender, type of cancer, comorbidities, surgical treatment history, chemotherapy history, and radiation treatment history were collected from medical records. Body mass index (BMI) was calculated by height and weight. Palliative performance scale (PPS) and palliative prognostic index (PPI) were interviewed. Phase angle was investigated at the time of admission with serum protein and albumin levels reflecting the basic nutritional status.

2) Evaluation of palliative performance scale and palliative prognostic index

PPS is a modified Karnofsky performance index that evaluates patients' performance status. It reflects five functional statuses: ambulation, degree of disease, self-care, oral intake, and consciousness. PPS ranged from $0 \%$ to $100 \%$ in $10 \%$ increment, with $100 \%$ defined as a normal and healthy state whereas $0 \%$ defined as death. ${ }^{14,15)}$

PPI, developed by Morita, could evaluate both performance status and the following clinical findings well-known to be associated with poor prognosis: dietary intake, edema, dyspnea on exertion, and delirium. ${ }^{16)}$ Performance status was scored from 0 to 4 by grouping Karnofsky performance index. Dietary intake ranged from 0 to 2.5 , edema from 0 to 1 , dyspnea on exertion from 0 to 3.5 , and delirium from 0 to 4 by the degree and presence of symptoms. The total score was the sum of the performance and clinical scores from the lowest 0 to the highest 15 points. 
3) Measurement of the phase angle by bioelectrical impedance analysis

BIA was measured using Biodynamics model 450 body composition analyzer (Biodynamics Co., Seattle, WA, USA). Patients dropped their legs in bed with supine state, and took a position with arms not touching the body. The two sensing electrodes were attached to the right side of the wrist and ankle. The two current electrodes were attached to the right dorsum of hand and foot. Data of sex, height, weight, and age were added to the machine which automatically calculated their values. ${ }^{17)}$ Resistance and reactance results were provided with a current of $800 \mu \mathrm{A}$ and BIA at $50 \mathrm{Khz}$. Phase angle was obtained using the following formula:

Phase angle $=($ resistance $/$ reactance $) \times(180 / \pi)$.

4) Statistical analysis

By using the frequency analysis, the continuous variables were described by the median and interquartile range. Categorical variables were described by the frequency and percentile. The correlation between the phase angle and PPS, PPI, phase angle, and survival time were analyzed using the Spearman correlation analysis. Univariate and multivariate Cox regression analysis were used to examine variables affecting the survival time of the study subjects. Differences of survival time of phase angle scores were analyzed by log-rank test. SPSS ver. 16.02 (SPSS Inc., Chicago, IL, USA) was used for all statistical analyses. Significance was defined if the probability (P-value) was less than 0.05 .

\section{RESULTS}

\section{Demographic and Clinical Characteristics}

Of 28 study subjects, 13 (46.4\%) were male patients and 15 (53.6\%) were females. The most common age group (53.6\%) were over 70 years old, followed by 50 to 70 years old (35.7\%), and 30 to 50 years old (10.7\%). The most common type of cancer was digestive tract cancer with 11 patients (39.2\%), including stomach, colon, digestive system, and liver cancer. The number of lung cancer, hematologic cancer, and or bladder/kidney cancer patients was 3 . Nine patients had diabetes. Five patients had hypertension. Of the 28 patients, 19 (67.8\%) underwent chemotherapy, $16(57.1 \%)$ received radiation therapy, and 12
(42.8\%) had surgery. The median score of BMI, PPI, PPS, and phase angle were $19.80 \mathrm{~kg} / \mathrm{m}^{2}$ (15.10-24.60), 4.5 (0-9), 50\% $(20,70)$, and $4.50^{\circ}(1.8-6.5)$, respectively. The median survival time of the patients was 25.5 days (78.5\%), with 22 patients expired at the end of the study (Table 1).

Table 1. Clinical characteristics of the patients

\begin{tabular}{|c|c|}
\hline Characteristic & Value \\
\hline \multicolumn{2}{|l|}{ Sex } \\
\hline Men & $13(46.4)$ \\
\hline Women & $15(53.6)$ \\
\hline \multicolumn{2}{|l|}{ Age (y) } \\
\hline$\leq 50$ & $3(10.7)$ \\
\hline $51-70$ & $10(35.7)$ \\
\hline$>70$ & $15(53.6)$ \\
\hline
\end{tabular}

Cancer type

Digestive tract $\quad 11(39.2)$

Lung $3(10.7)$

Hematology $3(10.7)$

Bladder/renal $\quad 3(10.7)$

Other $8(28.5)$

Comorbidities

Hypertension

Diabetes mellitus

Ever surgical treatment

12 (42.8)

Ever chemotherapy

19 (67.8)

Ever radiotherapy

$16(57.1)$

Height $(\mathrm{cm})^{*}$

$161.50(145-178)$

Weight (kg)*

$51.50(38-71)$

Body mass index $\left(\mathrm{kg} / \mathrm{m}^{2}\right)^{*, \dagger}$

$19.80(15.10-24.60)$

Protein (g/dL)*

$5.8(4.5-7.9)$

Albumin (g/dL)*

$2.7(1.7-3.9)$

Palliative prognostic index*

$4.5(0-9)$

Palliative performance scale (\%)*

$50(20-70)$

Phase angle $\left({ }^{\circ}\right)^{*}$

$4.50(1.8-6.5)$

Expired patients

22 (78.6)

Survival time (d)*

$25.5(6-126)$

Values are presented as number (\%) or median (inter quartile range). *Data are presented as median $(\mathrm{Q} 1, \mathrm{Q} 3) .{ }^{\dagger}$ Calculated as weight in kilograms divided by height in meters squared. 
2. Relationship between Phase Angle and Palliative Performance Scale, Palliative Prognostic Index, Body Mass Index, and Survival Time

We used Spearman's correlation analysis to evaluate the correlation between phase angle and PPS, PPI, BMI, and the survival time. Phase angle showed significant positive correlation $(\mathrm{r}=0.395, \mathrm{P}<0.05)$ with the survival time. BMI exhibited significant negative correlation $(\mathrm{r}=-0.538, \mathrm{P}<0.05)$ with PPI and positive correlation $(\mathrm{r}=0.493, \mathrm{P}<0.05)$ with survival time. PPI showed significant negative correlation $(\mathrm{r}=-0.408, \mathrm{P}<0.05)$ with the survival time (Table 2).

\section{Factors Affecting the Survival: Univariate Analysis}

We examined factors affecting the survival by univariate Cox regression analysis. Phase angle showed statistically significant predication of survival (hazard ratio [HR], 0.61 ; 95\% confidence

Table 2. Spearman's correlation coefficients of phase angle, BMI, PPS, PPI, and survival time

\begin{tabular}{lcccc}
\hline \multicolumn{1}{c}{ Variable } & Phase angle & BMI & PPS & PPI \\
\hline BMI & 0.318 & & & \\
PPS & 0.098 & 0.225 & & \\
PPI & -0.137 & $-0.538^{*}$ & -0.365 & \\
Survival time & $0.395^{*}$ & $0.493^{*}$ & 0.133 & $-0.408^{*}$ \\
\hline
\end{tabular}

Values are presented as correlation coefficient by Spearman's correlation analysis.

BMI: body mass index, PPS: palliative performance scale, PPI: palliative prognostic index.

*Shows P-value $<0.05$.

Table 3. Variables influenced on patients' survival by univariate analysis

\begin{tabular}{lcc}
\hline \multicolumn{1}{c}{ Variable } & $\begin{array}{c}\text { Hazard ratio } \\
(95 \% \text { confidence interval })\end{array}$ & P-value* $^{*}$ \\
\hline Phase angle & $0.61(0.42,0.89)$ & 0.010 \\
Age & $0.99(0.95,1.02)$ & 0.426 \\
Palliative performance scale & $0.99(0.97,1.02)$ & 0.732 \\
Palliative prognostic index & $1.21(1.00,1.47)$ & 0.048 \\
Body mass index & $0.86(0.74,1.00)$ & 0.053 \\
\hline
\end{tabular}

*Assessed by univariate Cox's regression analysis. interval $[\mathrm{CI}], 0.42-0.89 ; \mathrm{P}=0.01)$. PPI also showed significant predictive value for survival ( $\mathrm{HR}, 1.21$; 95\% CI, 1.00-1.47; $\mathrm{P}=$ 0.048). However, age, PPS, and BMI did not showed significant relationship with survival (Table 3 ).

\section{Factors Affecting the Survival: Multivariate Analysis}

We examined effects of age, PPI, BMI, and phase angle on survival by using the multivariate Cox regression analysis. Adjusted for age, PPI, and BMI, only phase angle showed statistically significant predictive value for survival (HR, 0.64; 95\% CI, 0.42-0.95; P = 0.03) (Table 4).

\section{Difference in Survival according to the Phase Angle}

We divided phase angle into two groups with cut-off value of

Table 4. Variables influenced on patients' survival by multivariate analysis

\begin{tabular}{lcc}
\hline Variable & Hazard ratio (95\% confidence interval) & P-value* $^{*}$ \\
\hline Age & $0.98(0.94,1.02)$ & 0.256 \\
PPI & $1.23(0.97,1.57)$ & 0.083 \\
BMI & $0.93(0.77,1.12)$ & 0.424 \\
Phase angle & $0.64(0.42,0.95)$ & 0.028 \\
\hline
\end{tabular}

BMI: body mass index, PPI: palliative prognostic index.

*Assessed by multivariate Cox's regression analysis. Multivariate models were adjusted for age, PPI, BMI, and phase angle.

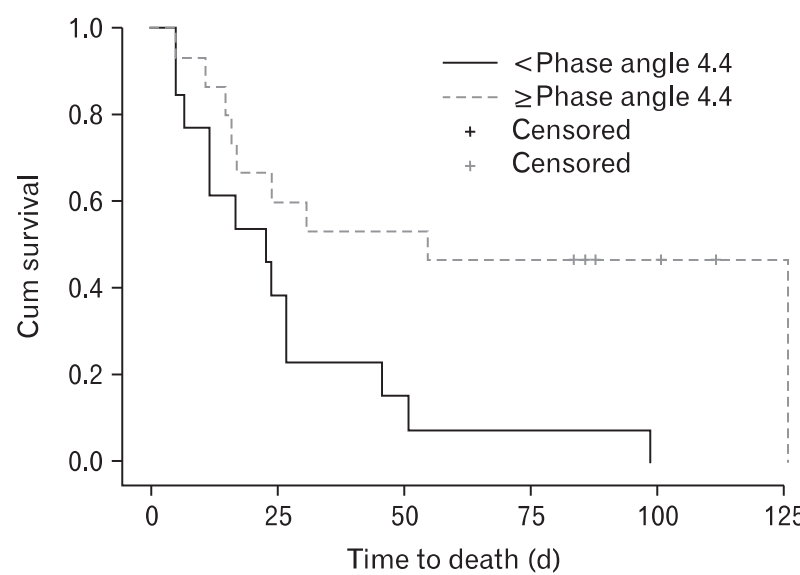

Figure 1. Kaplan-Meier survival analysis by phase angle group (logrank, 6.208; P-value = 0.013). Phase angle score below 4.4 showed significant shorter survival than phase angle score over 4.4. 
4.4 degree. Kaplan-Meier survival curve revealed that phase angle larger than $4.4^{\circ}$ showed statistically significant $(\mathrm{P}=0.01)$ longer survival time compared to phase angle less than $4.4^{\circ}$ (Figure 1).

\section{DISCUSSION}

Accurate survival prediction is essential for decision-making in palliative care to prevent unnecessary treatment. This study demonstrated that phase angle has significant prognostic value in terminal cancer patients. Phase angle score over 4.4 degree had longer survival time than those below 4.4 degree. Previous studies on phase angle also showed similar results. Toso et al. ${ }^{18)}$ reported that a significant longer survival in group of phase angle over 4.5 degree in lung cancer patients. Hui et al. ${ }^{19)}$ also showed longer survival time in advanced cancer patients with phase angle over 4.5 degree. Gupta et al. ${ }^{7-9)}$ reported that pancreatic cancer patients with median phase angle score over 5 degree and advanced colorectal cancer and breast cancer patients with over 5.6 degree showed longer survival time. Although these studies were performed in different populations, they provided the following consistent results: over a certain cutoff value, phase angle showed longer survival time. It is generally agreed that phase angle less than 5 degree will need medical management in any person.

Weight loss due to malnutrition, decrease in muscle strength, and decrease of quality of life are reported to be associated with shorter survival. ${ }^{20)}$ The mechanism of phase angle is probably originated from the predictability of nutritional status. Malnutrition can strongly affect the electric characteristics of human body. Phase angle calculated by resistance and reactance of body can demonstrate distribution of BMI, muscle mass, and dehydration status. ${ }^{21)}$ Moreover, phase angle affect both nutritional status and physical function or strength. ${ }^{22)}$ Body resistance and reactance could predict muscle strength associated with hand grip strength, maximal expiratory flow, and Karnofsky index in advanced cancer patients. ${ }^{13,23)}$ Our findings suggest that body fluid and cellular membrane status might predict prognosis as much as the pre-existing prognostic factor such as physical function and performance status. Future studies about cellular function, body fluid, and nutritional status are merited.

In this study, we compared prognostic value of phase angle with PPS and PPI, the well known prognostic factors in palliative care. To predict mortality within 6 month, PPS has a predictability of $96 \%$ at PPS $10 \%$ to $20 \%, 89 \%$ at $30 \%$ to $40 \%$, and $81 \%$ over $50 \% .{ }^{15)}$ PPI index more than 6 showed $80 \%$ sensitivity and $85 \%$ specificity mortality. ${ }^{16)}$ However, PPS could not present prognostic value in Cox regression analysis. PPI only showed prognostic value in univariate Cox regression analysis. This is probably due to the small number with minute discrimination of PPS score in each patient. In addition, co-linearity of PPS and PPI may have affected the result. To overcome the limitation of this study, a large scale study with longer observation period is needed.

This study has several limitations. Firstly, the small study population (28 subjects) may restrict the generalization on the outcome of phase angle. Further prospective study with various number and patients are recommended. Secondly, because this study is performed in tertiary hospital inpatients, we need information on outpatients and different medical facility. Finally, we excluded patients with edema, pleural effusion, and ascites in this study. These are frequent signs of terminal cancer patients. Therefore, this might have resulted in selection bias.

To our knowledge, this was the first study that evaluated the correlation between phase angle and survival in terminal cancer patients in Korea. Although the study had its limitations, our results demonstrated that phase angle could provide significant prognostic value for survival.

Phase angle probably have prognostic value in terminal cancer patients. Low phase angle score predicts poor prognosis.

\section{CONFLICT OF INTEREST}

No potential conflict of interest relevant to this article was reported.

\section{REFERENCES}

1. Inui A. Cancer anorexia-cachexia syndrome: current issues in research and management. CA Cancer J Clin 2002;52:72-91.

2. Barbosa-Silva MC, Barros AJ, Post CL, Waitzberg DL, Heymsfield SB. Can bioelectrical impedance analysis identify malnutrition in preoperative nutrition assessment? Nutrition 
2003;19:422-6.

3. Fredrix EW, Saris WH, Soeters PB, Wouters EF, Kester AD, von Meyenfeldt MF, et al. Estimation of body composition by bioelectrical impedance in cancer patients. Eur J Clin Nutr 1990;44:749-52.

4. Zarowitz BJ, Pilla AM. Bioelectrical impedance in clinical practice. DICP 1989;23:548-55.

5. Barbosa-Silva MC, Barros AJ. Bioelectrical impedance analysis in clinical practice: a new perspective on its use beyond body composition equations. Curr Opin Clin Nutr Metab Care 2005;8:311-7.

6. Kyle UG, Bosaeus I, De Lorenzo AD, Deurenberg P, Elia M, Manuel Gomez J, et al. Bioelectrical impedance analysis-part II: utilization in clinical practice. Clin Nutr 2004;23:1430-53.

7. Gupta D, Lis CG, Dahlk SL, Vashi PG, Grutsch JF, Lammersfeld CA. Bioelectrical impedance phase angle as a prognostic indicator in advanced pancreatic cancer. Br J Nutr 2004;92:957-62.

8. Gupta D, Lammersfeld CA, Burrows JL, Dahlk SL, Vashi PG, Grutsch JF, et al. Bioelectrical impedance phase angle in clinical practice: implications for prognosis in advanced colorectal cancer. Am J Clin Nutr 2004;80:1634-8.

9. Gupta D, Lammersfeld CA, Vashi PG, King J, Dahlk SL, Grutsch JF, et al. Bioelectrical impedance phase angle as a prognostic indicator in breast cancer. BMC Cancer 2008;8: 249.

10. Gupta D, Lammersfeld CA, Vashi PG, King J, Dahlk SL, Grutsch JF, et al. Bioelectrical impedance phase angle in clinical practice: implications for prognosis in stage IIIB and IV non-small cell lung cancer. BMC Cancer 2009;9:37.

11. Santarpia L, Marra M, Montagnese C, Alfonsi L, Pasanisi F, Contaldo F. Prognostic significance of bioelectrical impedance phase angle in advanced cancer: preliminary observations. Nutrition 2009;25:930-1.

12. Paiva SI, Borges LR, Halpern-Silveira D, Assuncao MC, Barros AJ, Gonzalez MC. Standardized phase angle from bioelectrical impedance analysis as prognostic factor for survival in patients with cancer. Support Care Cancer 2010; 19:187-92.

13. Norman K, Stobaus N, Zocher D, Bosy-Westphal A, Szramek
A, Scheufele R, et al. Cutoff percentiles of bioelectrical phase angle predict functionality, quality of life, and mortality in patients with cancer. Am J Clin Nutr 2010;92:612-9.

14. Anderson F, Downing GM, Hill J, Casorso L, Lerch N. Palliative performance scale (PPS): a new tool. J Palliat Care 1996;12:5-11.

15. Harrold J, Rickerson E, Carroll JT, McGrath J, Morales K, Kapo J, et al. Is the palliative performance scale a useful predictor of mortality in a heterogeneous hospice population? J Palliat Med 2005;8:503-9.

16. Morita T, Tsunoda J, Inoue S, Chihara S. The Palliative Prognostic Index: a scoring system for survival prediction of terminally ill cancer patients. Support Care Cancer 1999;7: 128-33.

17. Lukaski HC, Johnson PE, Bolonchuk WW, Lykken GI. Assessment of fat-free mass using bioelectrical impedance measurements of the human body. Am J Clin Nutr 1985;41: 810-7.

18. Toso S, Piccoli A, Gusella M, Menon D, Bononi A, Crepaldi $\mathrm{G}$, et al. Altered tissue electric properties in lung cancer patients as detected by bioelectric impedance vector analysis. Nutrition 2000;16:120-4.

19. Hui D, Bansal S, Morgado M, Dev R, Chisholm G, Bruera E. Phase angle for prognostication of survival in patients with advanced cancer: preliminary findings. Cancer 2014;120: 2207-14.

20. Tisdale MJ. Cancer cachexia. Curr Opin Gastroenterol 2010; 26:146-51.

21. Norman K, Smoliner C, Kilbert A, Valentini L, Lochs H, Pirlich M. Disease-related malnutrition but not underweight by $\mathrm{BMI}$ is reflected by disturbed electric tissue properties in the bioelectrical impedance vector analysis. Br J Nutr 2008; 100:590-5.

22. Selberg O, Selberg D. Norms and correlates of bioimpedance phase angle in healthy human subjects, hospitalized patients, and patients with liver cirrhosis. Eur J Appl Physiol 2002;86: 509-16.

23. Norman K, Pirlich M, Sorensen J, Christensen P, Kemps M, Schutz T, et al. Bioimpedance vector analysis as a measure of muscle function. Clin Nutr 2009;28:78-82. 